\title{
The Use of Ovarian Wall as an Endobag During Laparoscopic Removal of a Giant Dermoid Cyst: A New Approach
}

\author{
Zahra Alamooti ${ }^{1}$, Lakshmi Bayi Sushama $^{*}$ (D)
}

\begin{abstract}
Objectives: Incidental finding of an asymptomatic giant ovarian mass is unusual during pregnancy. Addressing such voluminous cysts laparoscopically is controversial, and a challenge to the surgeon. This article describes on how we tackled a 27-cm ovarian cyst using the ovarian wall itself as the endobag.

Case Presentation: A 32-year-old lady was referred to Obstetrics and Gynecology Department at 9 weeks of gestation with missed miscarriage and an incidental finding of a huge ovarian cyst by ultrasonography. After evaluation and obtaining informed consent, laparoscopy was performed. We elaborate here on how this huge mass was excised laparoscopically, minimizing intraperitoneal spillage by using the ovarian wall itself as endobag. The histopathology report confirmed a mature cystic teratoma. The postoperative period was uneventful.

Conclusions: Many surgeons have a phobia to tackle giant ovarian cysts laparoscopically, due to fear of malignancy and spillage of contents, despite the inherent advantages of the minimal access surgery. We hope that this case report will add to the increasing evidence on the safety and efficacy of tackling such giant cysts laparoscopically.

Keywords: Giant ovarian cyst, Dermoid cyst, Ovarian wall, Endobag, Laparoscopy
\end{abstract}

\section{Introduction}

Asymptomatic adnexal masses are diagnosed incidentally in $4 \%$ of pregnancies (1). Majority of them are benign and are of ovarian origin (1). Giant cysts, though uncommon, are challenging to the surgeon, in their removal. Such huge cysts were conventionally managed by laparotomy. In spite of all the advantages inherent to minimal access surgery, many surgeons refrain from removing them laparoscopically due to the risk of malignancy and the spillage of contents. This is a case report where a giant benign cystic teratoma (dermoid cyst) of $27 \mathrm{~cm}$ size was successfully excised laparoscopically. The greatest challenge was the retrieval of the huge specimen which would not fit into the largest endobag. The cyst was successfully removed using the ovarian wall as the endobag, which curtailed peritoneal soiling by the intra-cystic contents. We hope that this paper will add to the emerging evidence on the safety of laparoscopy in removing such gigantic cysts, refuting the myth that spillage of contents and its complications increases in proportion with cyst size. To the best of our knowledge, this is the largest reported ovarian cyst, removed laparoscopically in Qatar.

\section{Case Presentation}

The patient was a 32-year-old woman Gravida 3 Para 2 Live 2, who was referred to Obstetrics and Gynecology
Department at 9 weeks of gestation with missed miscarriage and an incidental finding of a huge $21-\mathrm{cm}$ ovarian cyst by ultrasonography. She was asymptomatic and was diagnosed to have this ovarian cyst during her viability scan at 7 weeks of gestation.

On evaluation, she had no risk factors suggestive of a malignant potential like loss of appetite, abdominal bloating, loss of weight, dyspepsia or family history of breast/ gastrointestinal/ ovarian cancer. Physical examination showed a pelvic mass occupying the hypogastrium, bilateral iliac quadrants and reaching up to the umbilicus. The mass was not tender and there was no ascites, pleural effusion, palpable lymph nodes or hepatomegaly. No pressure symptoms like varicose veins were present. A repeat abdominopelvic ultrasound showed a $21-\mathrm{cm}$ adnexal multi-locular cyst/cystadenoma with no solid component. The tumor markers CA-125, serum alpha-fetoprotein (AFP), serum lactate dehydrogenase (LDH), carcinoembryonic antigen (CEA) were tested and found to be within normal limits.

The management options, both laparotomy and laparoscopic surgery, were discussed with the patient, including their pros and cons. Patient opted for laparoscopic removal as her primary concern was to avoid a large abdominal scar. Informed consent was obtained, and she was posted for elective operative laparoscopy after 


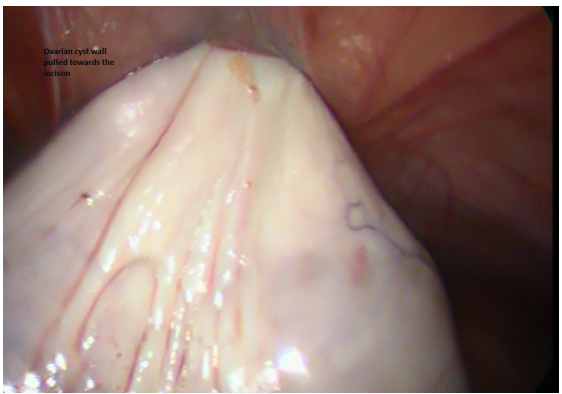

Figure 1. Ovarian Cyst Wall Used as a Substitute to Endobag and Being Pulled Towards Incision.

anesthetic assessment.

\section{Procedure}

Suction evacuation of the missed miscarriage was done. Abdominal cavity was entered through 3 ports (one 10 $\mathrm{mm}$ sited $1 \mathrm{~cm}$ above the umbilicus and two $5 \mathrm{~mm}$ ) after an uneventful pneumoperitoneum.

Findings at laparoscopy: Left ovary was enlarged to 27 $\mathrm{cm}$. Ovarian wall was smooth in outline with no papillary projections. There was no differentiation between the normal ovarian tissue and the cyst. The left Fallopian tube was stretched over the cyst. Left broad ligament was free. The uterus, the right ovary and the right Fallopian tube were normal. There was no ascites. Inspection of the paracolic gutters, mesentery, surface of intestines, paraaortic lymph nodes, liver and spleen and sub-phrenic spaces were normal.

The ovarian mass was stabilized with the help of a grasper, and the cyst was punctured. Around $2 \mathrm{~L}$ of liquid sebaceous material was aspirated and the cyst wall was gradually collapsed revealing multiloculations and hair within the cyst. Left salpingo-oophorectomy was done by cauterizing and cutting the left ovarian pedicle. The cyst and its remaining contents would not fit into any endoscopic bag. To prevent the hair and other debris within the cyst from soiling the peritoneal cavity, the redundant cyst wall was pulled over to serve as an endobag using graspers (Figure 1). The crux of the procedure lay in maneuvering the cyst (with its contents), to be exenterated through a $2-\mathrm{cm}$ suprapubic incision, taking care not to let down the edges of the open cyst wall, as, that may account for spillage of cyst contents. Despite our best efforts, there was minimal spill of sebaceous material into the Pouch of Douglas, which was meticulously aspirated, followed by peritoneal lavage. After exteriorizing the cyst, the inner wall of the cyst was examined to rule out features suggestive of malignant potential (like papillary excrescences, solid areas with foci of haemorrhage and necrosis). Total operating time from start of the procedure was 2 hours and 18 minutes. The blood loss at surgery was minimal.

The patient had an uneventful post-operative period and was discharged on the second day of surgery. She had post-operative review after 2 weeks. The histopathology report confirmed dermoid cyst, with no evidence of malignancy. Histopathology of the suction evacuation specimen was consistent with the products of conception with no evidence of trophoblastic disease.

\section{Discussion}

Incidental adnexal masses are diagnosed in $4 \%$ of pregnancies (1). Majority of them are benign and of ovarian origin (1). Most of these ovarian cysts seen during pregnancy are follicular /lutein cyst that resolve during pregnancy or are due to benign conditions, such as benign cystic teratomas (as in our case) or endometriomas $(1,2)$. Benign mature teratomas or dermoid cysts, arising from the "totipotential cell rests" in the ovary, have an ability to differentiate into the tissues that may represent all layers (ectoderm, endoderm and mesoderm), though one layer predominates. In our case, there were tissues from the ectodermal layer, hair and sebum, which predominated. Dermoid cysts constitute $20 \%-25 \%$ of ovarian tumors $(3,4)$, and account for up to $70 \%$ of benign ovarian neoplasms occurring in women of the childbearing years (15-45 years) (5).

Laparoscopy is the standard treatment for excision of benign ovarian cysts and provides many advantages over laparotomy (4-12). Though laparoscopic surgery is associated with a longer operating time compared to laparotomy, the advantages are: less postoperative pain, reduced postoperative analgesic need, earlier mobilization which reduces chances of deep venous thrombosis, short hospital stay, reduced infectious morbidity, earlier recovery, better quality of life with early return to normal activity during the postoperative period and acceptable mini incisions (3-5,8-16). Because most patients with benign cystic teratomas belong to the reproductive age, a minimally invasive approach is ideal, as it minimizes the risk of adhesions and its effects. Peritubal adhesions may compromise fertility, whereas peritoneal adhesions can cause intestinal obstruction $(3,4)$.

Major constraints to the laparoscopic treatment of ovarian cysts are:

- the size of the cyst,

- expertise of the surgeon and technical difficulties,

- the fear of undiagnosed malignancy,

- $\quad$ spillage of contents and its late complications, and

- retrieval of a relatively large specimen through a mini-incision.

Regarding the Size of the Cyst

There is no standard cut off measurements to define a large ovarian cyst by its size (9). According to some authors, large ovarian cysts are those over $10 \mathrm{~cm}$ in diameter $(9,12,17)$, while others define it, as those cysts that reach above the umbilicus $(9,18)$. Few others term them as giant or voluminous when they are over $15 \mathrm{~cm}$ (16). Laparoscopic excision of ovarian cysts is the gold 
standard of care in women of child-bearing age $(9,10)$. Laparoscopic management of large ovarian masses, however, is still under debate $(5,10,16,18)$. Conventionally, these huge masses were dealt with by open laparotomy (8$11,13)$. There is increasing evidence that the vast majority of large adnexal masses can be resected laparoscopically $(12,14,16-20)$. With proper pre-operative evaluation and patient selection, the size of an ovarian cyst is no longer a contraindication for laparoscopic excision $(8,10,11,16)$.

Regarding Surgical Skills and Technical Difficulties The major determinants deciding the mode of surgery, whether by a traditional open laparotomy or by laparoscopy, are the nature of the mass, the operator experience and patient preference $(1,5)$. Technical difficulties in tackling such giant cysts, laparoscopically, include: difficulty in inserting the Veress needle/trocar, resulting in cyst puncture and spillage, and the technical challenge of a large specimen retrieval with minimal spillage through a mini incision $(10,13)$.

\section{Malignant Potential}

Presence of undiagnosed malignancy in an apparently benign ovarian cyst is a paramount reason for large cysts to be removed by open laparotomy. However, the incidence of ovarian cancer in pregnant women with adnexal mass is rare and ranges from $0.004 \%-0.04 \%$ (1). The risk of malignancy in giant ovarian teratoma is $0.61 \%$ (19). Even if these tumors turn out to be malignant, they are usually in borderline, which remain confined to the ovary and have very good prognosis $(1,2)$. The value of markers like serum AFP, Serum beta-hCG (human chorionic gonadotrophin), inhibin levels and Serum CA125 levels are compromised as they may be raised due to either placental or decidual cell production (1).

\section{Spillage of Contents}

There is conflicting evidence regarding the relationship between the cyst size and its risk of spillage of the contents. Some studies have found no relation between the two $(4,10$, 13), while others quote a higher probability of spillage with increasing cyst size $(3,5,13)$. Laparoscopic removal of dermoid cysts reports spillage rates ranging from $15 \%-$ $100 \%$, compared with $4 \%-13 \%$ via laparotomy $(3,4,13)$. Some have found that spillage rates are comparable in laparoscopy and laparotomy (15). Laparoscopic excision and spill could be further compromised by the surgical expertise to shrink the cyst by careful aspiration of intracystic contents avoiding spillage and exteriorizing the cyst wall and its content preferably using endobag. Spillage is remarkably less when specimen is retrieved within endobags $(3-7,10,13,20)$. Use of endobags reduces intracystic content spillage by four fold.

In our case, where the specimen was larger than the largest available endoscopic retrieval bags, the thick cyst wall of the dermoid, itself served as a substitute for

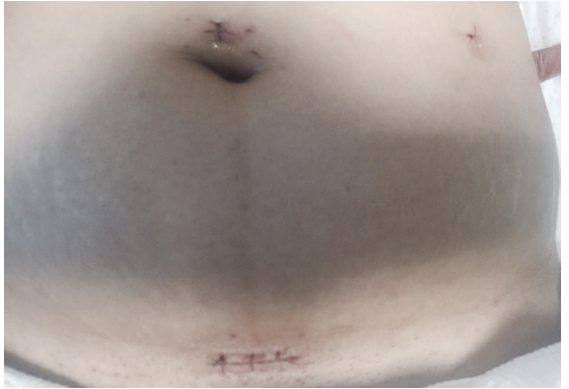

Figure 2. Post-surgery Wound.

endobag. This was possible because, the size of the cyst collapsed after aspiration of the intra-cystic sebaceous material, and the wall of the cyst was thick and smooth in outline, which facilitated the clasping of the cyst wall edges by a toothed grasper. Such a technique can be used only with ovarian cysts with relatively thick wall like endometriomas and dermoid cysts. This will not be possible in thin walled cyst, as the use of toothed grasper for better grasping of tissues, may rupture the cyst causing spillage of its contents.

Spillage of cyst contents is related with complications like pseudomyxoma peritonei (with mucinous cystadenoma), chemical peritonitis (with dermoid cyst) and the potential dissemination in the event of a rare occurrence of malignancy (13). Chemical peritonitis is an infrequent complication associated with dermoid cyst spillage $(3-5,12,13)$. The incidence is between $0.2 \%$ $8 \%$ and can result in pelvic adhesive disease/ peritubal adhesions $(3-5,13)$. There is emerging evidence that there is no significant difference in complication rates regardless of cystic spillage $(3-5,7)$. The risk of chemical peritonitis can be minimized during laparoscopy by meticulous jet lavaging of the peritoneal cavity $(3-5,7,8,16)$. In fact, laparoscopic approach allows better exposure and lavage of POD, which is the commonest site of collection of spilled content $(4,5,7)$.

\section{Retrieval of Specimen}

Specimen extraction in laparoscopic surgery is more wearisome than that in open laparotomy. In addition, tissue removal must be complete with minimal time limits to ensure the overall laparoscopic surgical advantages $(8,13)$. Specimen is usually extracted through a mini lap incision or through a posterior colpotomy. Solid specimen retrieval could be aided by tissue morcellators within endobags (13). In our case, we managed to remove the huge specimen through a small $2 \mathrm{~cm}$ suprapubic incision (Figure 2).

\section{Conclusions}

The size criteria and route of excision of large ovarian cysts are still a matter of debate. A vast majority of large adnexal masses are being resected laparoscopically with very minimal occurrence of presumed complications of cyst 
spillage and tumor dissemination. With proper patient selection, surgical expertise in gynecologic endoscopy, and advanced technology, laparoscopic excision of mammoth ovarian cyst is a reality and a dream come true with the inherent advantages of minimal access surgery.

\section{Acknowledgment}

We are really thankful to the anesthetic and nursing staff in the operation theatre for their professional support. We also extend our thanks to Dr. Sujjanna.A.L. Manuel, our colleague obstetrician and gynecologist, and Dr.I.P.Yadev for their kindness in sparing their valuable time for suggestions on correcting the draft.

\section{Ethical Issues}

The authors obtained informed consent from the patient for data collection and publication.

\section{Conflict of Interests}

We hereby declare that there is no conflict of interests.

\section{Financial Support}

None.

\section{References}

1. Spencer CP, Robarts PJ. Management of adnexal masses in pregnancy. Obstet Gynaecol. 2006; 8:14-19.

2. Condous G, Khalid A, Okaro E, Bourne T. Should we be examining the ovaries in pregnancy? Prevalence and natural history of adnexal pathology detected at firsttrimester sonography. Ultrasound Obstet Gynecol. 2004(1); 24:62-66.

3. Nezhat CR, Kalyoncu S, Nezhat CH, Johnson E, Berlanda N, Nezhat F. Laparoscopic Management of Ovarian Dermoid Cysts: Ten Years' Experience. JSLS. 1999; 3(3):179-184. doi: 10.1016/s0029-7844(99)90173-4

4. Shawki O, Soliman I, Ebrashy A, Sadek M,Bahnassy A.Laparoscopic management of ovarian dermoid cysts. Middle East Fertility Society Journal. 2004;9(1):58-65

5. Sinha A, Ewies AA. Ovarian Mature Cystic Teratoma: Challenges of Surgical Management. Obstet Gynecol Int. 2016; 2016: 2390178. doi: 10.1155/2016/2390178

6. Nezhat C, Winer WK, Nezhat F. Laparoscopic removal of dermoid cysts. Obstet Gynecol. 1989 Feb 1;73(2):278-81
7. Godinjak Z, Bilalović N, Idrizbegović E. Laparoscopic treatment of ovarian dermoid cysts is a safe procedure. Bosnian Journal of Basic Medical Sciences. 2011; 11(4):245-247.

8. Quinlan DK. The laparoscopic management of large ovarian cysts. J Obstet Gynaecol India. 2010;60(2):152-156

9. Alobaid A, Memon A, Alobaid S, Aldakhil L. Laparoscopic management of huge ovarian cysts. Obstet Gynecol Int. 2013; 2013:380854. doi: 10.1155/2013/380854

10. Vlahos NF, Iavazzo C, Marcopoulos MC, et al. Laparoscopic Management of Large Ovarian Cysts. Surg Innov. 2012;19(4) 370-374. doi: 10.1177/1553350611432722.

11. Sagiv R, Golan A, Glezerman M. Laparoscopic management of extremely large ovarian cysts. Obstet Gynecol. 2005;105(6):1319-1322. doi: 10.1097/01. AOG.0000159690.18634.f0

12. Mahmoud MS. The laparoscopic assisted management of a giant abdominopelvic ovarian mass. BAOJ Gynaecology. 2015; 1: 00113.

13. Stavroulis A, Memtsa M, Yoong W. Methods for specimen removal from the peritoneal cavity after laparoscopic excision. Obstet Gynaecol. 2013;15:26-30.

14. Howard FM. Surgical management of benign cystic teratoma. Laparoscopy vs. laparotomy. J Reprod Med. 1995; 40(7):495-9.

15. Yuen PM, Yu KM, Yip SK, Lou WC, Rogers MI, Chang A. A randomized prospective study of laparoscopy and laparotomy in the management of benign ovarian masses. Am J Obstet Gynecol. 1997;177(1):109-114.

16. Ye L-Y, Wang J-J, Liu D-R, Ding G-P, Cao L-P. Management of giant ovarian teratoma: A case series and review of the literature. Oncol Lett. 2012; 4(4):672-676. doi: 10.3892/ ol.2012.793

17. Ou CS, Liu YH, Zabriskie V, Rowbotham R. Alternate methods for laparoscopic management of adnexal masses greater than $10 \mathrm{~cm}$ in diameter. J Laparoendosc Adv Surg Tech A. 2001; 11(3):125-132.

18. Salem HAF. Laparoscopic excision of large ovarian cysts. J Obstet Gynaecol Res. 2002; 28: 290-294.

19. Ghezzi F, Cromi A, Bergamini V, et al. Should adnexal mass size influence surgical approach? A series of 186 laparoscopically managed large adnexal masses. BJOG. 2008;115(8):1020-1027.

20. Sunoo CS.Laparoscopic removal of a large adnexal mass. Obstet Gynecol. 2004;103(5 Pt 2):1087-1089. doi: 10.1097/01.AOG.0000117086.08661.3c

(c) 2018 The Author (s); This is an open-access article distributed under the terms of the Creative Commons Attribution License (http://creativecommons.org/licenses/by/4.0), which permits unrestricted use, distribution, and reproduction in any medium, provided the original work is properly cited. 